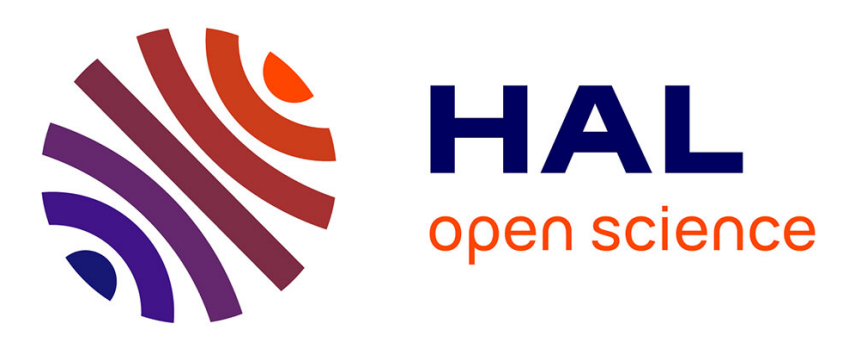

\title{
Comparison between direct numerical simulations and effective models for fluid-porous flows using penalization
}

Charles-Henri Bruneau, Didier Lasseux, Francisco J Valdés-Parada

\section{To cite this version:}

Charles-Henri Bruneau, Didier Lasseux, Francisco J Valdés-Parada. Comparison between direct numerical simulations and effective models for fluid-porous flows using penalization. Meccanica, 2020, 55 (5), pp.1061-1077. 10.1007/s11012-020-01149-7 . hal-03042108

\section{HAL Id: hal-03042108 https://hal.science/hal-03042108}

Submitted on 5 Dec 2020

HAL is a multi-disciplinary open access archive for the deposit and dissemination of scientific research documents, whether they are published or not. The documents may come from teaching and research institutions in France or abroad, or from public or private research centers.
L'archive ouverte pluridisciplinaire HAL, est destinée au dépôt et à la diffusion de documents scientifiques de niveau recherche, publiés ou non, émanant des établissements d'enseignement et de recherche français ou étrangers, des laboratoires publics ou privés. 


\title{
Comparison between direct numerical simulations and effective models for fluid-porous flows using penalization
}

\author{
Charles-Henri Bruneau ${ }^{1}$, Didier Lasseux ${ }^{2}$, Francisco J. Valdés-Parada ${ }^{3}$ \\ Corresponding author: bruneau@math.u-bordeaux.fr
}

${ }^{1}$ Univ. Bordeaux, CNRS, Bordeaux INP, IMB, UMR 5251, F-33405, Talence, France

INRIA, IMB, UMR 5251, F-33405, Talence, France

${ }^{2}$ CNRS, I2M, UMR 5295, Esplanade des Arts et Métiers, 33405 Talence, France

3 Departamento de Ingeniería de Procesos e Hidráulica, División de Ciencias Básicas e

Ingeniería, Universidad Autónoma Metropolitana-Iztapalapa, Av. San Rafael Atlixco 186, 09340, CDMX, Mexico

\begin{abstract}
This work is devoted to a numerical study of two-dimensional incompressible flows in a fluid-porous medium system placed on an impermeable wall. Flow in the whole system is studied either at the pore scale or at the Darcy scale (using a one-domain approach) and the models at both scales are solved with a penalization method using the same formal Navier-Stokes equations modified by a Darcy-like term. Several effective medium penalized models are considered for the simulations. Various flow regimes are investigated ranging from laminar to turbulent. The velocity profiles inside and outside the porous medium show significant discrepancies between the different penalization models compared to the direct numerical simulations. This work motivates further studies about the spatial variations of the penalization coefficient to be introduced in the effective medium models in order to better reproduce the physics near the fluid-porous medium boundaries.
\end{abstract}

Keywords: Fluid-porous media, Direct numerical simulation, Penalization method, Effective medium models.

\section{Introduction}

Momentum transport between a porous medium and a fluid has been a topic of intense research interest over, at least, half a century, starting with the pioneering theoretical and experimental study by Beavers and Joseph (BJ) [6], who analyzed creeping flow in a channel partially filled with a porous medium. These authors proposed to couple Darcy's law with the Stokes equation by means of a Robin-type matching boundary condition. Shortly after, Saffman [36], used a statistical approach to extend Darcy's law outside of the porous medium bulk. Then, the boundary condition is recovered from a boundary-layer analysis imposing a step function for the spatial variations of the permeability and porosity. More recently, this boundary condition has been derived using the homogenization technique [20] and the volume-averaging method [43]. However, numerical simulations performed at the pore-scale have shown that the slip velocity in the BJ model is extremely sensitive to the position of the fluid-porous medium boundary [26, 27].

One alternative to the use of the BJ model is to match the Darcy-Brinkman and Stokes equations by means of two boundary conditions. In this regard, Ochoa-Tapia and Whitaker [34] proposed that the velocity should be continuous (which contrasts with the BJ statement) and that there should be a jump in the viscous stress at the boundary. These ideas may be viewed as an extension of those by Neale and Nader [32] who used continuity conditions of both the velocity and stress in order to couple the Darcy-Brinkman model to Stokes equations. Other alternatives of matching conditions between these two equations have been proposed. For example, Cieszko and Kubik [16] used two linearly-independent BJ-type equations to carry out the matching, which may be interpreted as a discontinuity on both the velocity and the stress. However, a point against these works is that the validity of the Darcy-Brinkman model is questionable, in general, as pointed out by Auriault [5]. 
The above works can be classified as two-domain approaches because they rely on the matching between the governing equations in the porous medium with those in the fluid by means of convenient boundary conditions. However, from the above references, it appears that no definitive answer has been proposed in this modeling approach. Conversely, the one-domain approach makes use of a single average equation, with position-dependent coefficients, which can be used to model transport in the porous medium, the fluid and in the transition zone [4]. This has the advantage that no boundary conditions are required at the fluid-porous interface, at the expense of requiring knowledge of the spatial variations of the effective-medium coefficients such as the permeability (in the case of creeping flow) or the apparent-permeability (in the case of inertial or even turbulent flow). The prediction of these spatial variations is still an active research field in which sharp [21] or smooth [42] variations of the permeability have been proposed for creeping flow conditions. Furthermore, the one-domain approach can be used in conjunction with the differential equations in the two-domain approach to derive the corresponding boundary conditions [34] and to predict their coefficients [42]. For this reason, the one-domain approach is used in this work.

Literature on flow between a porous medium and a fluid has been mainly dedicated so far to creeping flow in a channel partially filled with a porous medium (BJ configuration) and extensions to the inertial (or turbulent) regime are still scarce. A complete one-domain averaged (but unclosed) model valid everywhere and including inertia was derived in [33] and recently revisited in [35] (see Eqs. (17) and (18) therein). In the former reference, a closure scheme was proposed for a two-domain approach while ignoring inertia in the homogeneous part of the porous region. The unclosed one-domain model could be used here as this is the most complete description available so far. Nevertheless, the solution of this model is still an important challenge as it requires to predict the porosity gradient in the interfacial region and the pore-scale pressure and velocity fields to close the model. This is obviously not a computational procedure which can be envisaged as a routine solution to the problem. An alternative to circumvent this difficulty is to use an empirical closure scheme as proposed in $[8,9,37]$ for the BJ configuration. In these works, an empirical form of the spatial variation of the porosity was assumed. It was used in empirical correlations between the porosity and the permeability and inertial resistance coefficient usually employed for the homogeneous part of the porous medium under consideration.

Our purpose in the present work is to investigate whether an effective-medium model, derived for a homogeneous porous medium (i.e. far from the interfaces with a fluid region), which is much simpler than the complete one-domain averaged model, remains accurate when employed in the whole domain using a penalization approach. This effective-medium model can be formally viewed as a Navier-Stokes equation written in terms of spatial averages of the velocity and pressure with addition of a Darcy-like penalization term. This is performed on a system different from the BJ configuration with the idea that, in many practical situations, the mean flow is not fully developed along the fluid-porous medium interface and that this interface is not necessarily parallel to the mean flow. The configuration under consideration here is similar to those used to study bluff bodies [1]. It is made of a two-dimensional rectangular porous slab consisting of a square pattern of parallel cylinders immersed in a semi infinite fluid region limited by a solid wall. The aim is first to accurately compute the incompressible flow orthogonal to the cylinders' axes inside and outside the porous medium. This is achieved by a Direct Numerical Simulation (DNS) of the penalized Navier-Stokes equations. The DNS serves as a reference for further comparisons with the effective homogeneous model and has archival value for subsequent studies in this particular configuration. In a second step, the porous zone is considered as a homogeneous medium taking into account its properties, namely, the permeability coefficient and porosity. Three different forms of the Darcy-like penalization term in the effective-medium model are investigated. Ultimately, the purpose is to show that the flow solution for a fluid-porous system obtained with the effective model using a penalization approach on a coarse grid is much faster than with DNS.

Both DNS and the solution of the effective medium models are performed using a penalization approach as it will be explained in further details below. An appealing feature of this approach is that the formulation of the momentum equations is the same (although pressure and velocities do not have the same scale for the DNS and effective medium simulations). The only difference lies in the way penalization is applied to the Darcy-like term. The penalized equations are approximated by an accurate finite differences scheme and solved using a multigrid procedure involving several grid levels. The code is highly parallelized with Message Passing Interface (MPI) directives.

Models and results are carefully analyzed to determine which effective-medium model yields results 


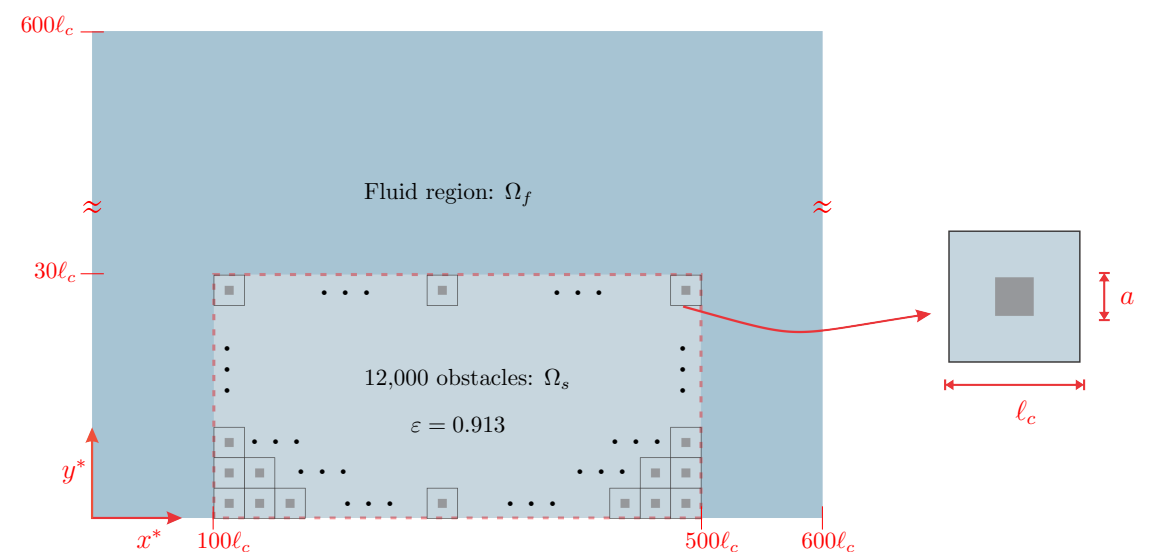

a)

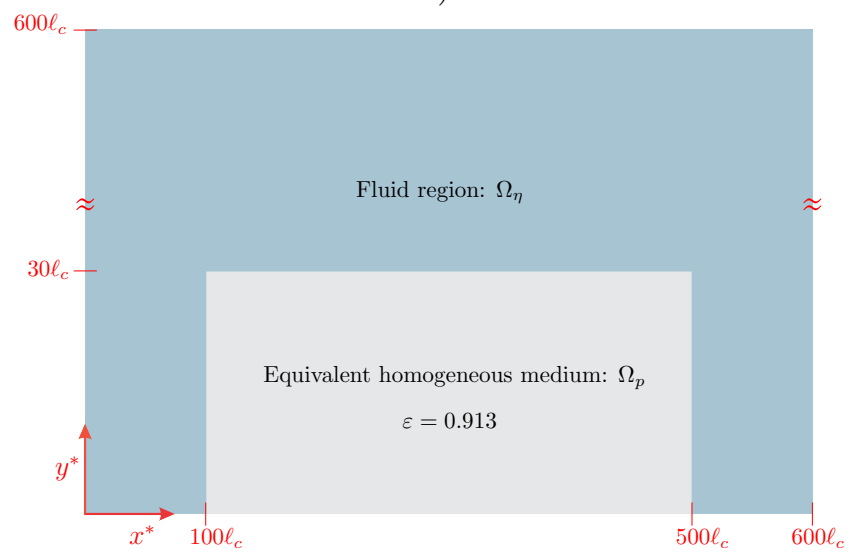

b)

Figure 1: Domain configuration for a) the DNS and b) the effective medium approach.

closest to those obtained from DNS. In particular the flow fields inside the porous medium are scrutinized to investigate the relevance of the different effective medium models close to and far from the porous zone.

The following sections are organized as follows. In section 2, the pore scale and heuristic effective medium models are reported. The use of the penalization procedure for both models is explained. Section 3 is dedicated to the description of the numerical method. The numerical results corresponding to DNS are compared with those obtained with the effective medium approach in section 4 . In Appendix B, it is shown that some improvement to the effective medium approach can be obtained empirically. Conclusions are provided in section 5 .

\section{Modeling}

The aim of this work is to compare as accurately as possible the DNS of an unsteady two-dimensional Newtonian one-phase flow inside and around a porous medium to heuristic versions of effective medium models using the penalization approach. To do so, consider a domain $\Omega$ partially filled with a regular pattern of in-line parallel cylinders of square cross-section as the one depicted in Fig. 1. The pattern is obtained from $400 x^{*}$ - and $30 y^{*}$-periodic repetitions of a unit cell of size $\ell_{c} \times \ell_{c}$ (see Fig. 1a).

The solid cylinders $\Omega_{s}$, have an edge size $a=0.295 \ell_{c}$, yielding a porosity of the unit cell $\epsilon=0.913$. The entire domain, saturated by the fluid occupying the region $\Omega_{f}$, has a size $600 \ell_{c} \times 600 \ell_{c}$ and the porous zone, corresponding to the portion occupied by the cylinder pattern, is placed at $100 \leqslant x^{*} / \ell_{c} \leqslant 500$, $0 \leqslant y^{*} / \ell_{c} \leqslant 30$. The lower wall is impermeable, while the vertical size of the domain is large enough to avoid the influence of the upper boundary condition due to the blockage as shown in [15]. The flow approaches the 
system from the left boundary with a constant velocity $\mathbf{v}=u_{\infty} \mathbf{e}_{x}$, while at the exit, on the right boundary, the traction is specified following a formulation provided in [12].

The aim is first to compute a reference flow using the DNS around the 12,000 squares by solving the penalized Navier-Stokes equations for the genuine unknowns velocity $\mathbf{u}$, and pressure $p$, in the fluid domain $\Omega_{f}$. For the sake of simplicity and in order to use a formulation that is uniform for both the DNS and the computation of the effective model, the flow is solved in the whole domain $\Omega$ using the penalization method $[2,25,10]$. This allows to easily handle the 12,000 solid squares without the need of an adapted mesh. The advantages and disadvantages of this method are discussed in detail in[31]. It should be pointed out that the volume (or Brinkman) penalization method has been extensively used over the past twenty years to solve problems in complex geometries [10]. It is particularly suited for all kind of spectral or finite differences approximations $[24,23]$ but is also used with other approximations [39, 30, 40]. The method is applied directly to simulate incompressible flows or is extended to other situations including moving solid obstacles [22] and heat transfer [3] for instance. The aim is always to easily handle fixed or moving complex geometries $[19,38,18,7]$. Authors found agreement with laboratory experiments or with benchmark results obtained with unstructured meshes.

Under these circumstances, using $\ell_{c}, u_{\infty}, \ell_{c} / u_{\infty}$ and $\rho u_{\infty}^{2}$ as the references for the lengths, velocity, time and pressure, respectively, the model can be written in the dimensionless form as

$$
\begin{aligned}
& \partial_{t} \mathbf{u}+\mathbf{u} \cdot \nabla \mathbf{u}=-\nabla p+\frac{1}{R e} \nabla^{2} \mathbf{u}-\boldsymbol{\Gamma} \cdot \mathbf{u} \\
& \nabla \cdot \mathbf{u}=0 \\
& \text { B.C.1 } \mathbf{u}=\mathbf{0} \\
& \text { B.C. } 2 \nabla \mathbf{u} \cdot \mathbf{n}=0 \\
& \text { B.C. } 3 \mathbf{u}=\mathbf{e}_{x} \\
& \text { B.C.4 } \boldsymbol{\Sigma} \cdot \mathbf{n}+\frac{1}{2}(\mathbf{u} \cdot \mathbf{n})^{-}\left(\mathbf{u}-\mathbf{u}_{r e f}\right)=\boldsymbol{\Sigma}_{r e f} \cdot \mathbf{n} \\
& \text { I.C. } \mathbf{u}=\mathbf{0} \\
& \begin{array}{r}
\text { in } \Omega_{T} \\
\text { in } \Omega_{T} \\
\text { at } 0 \leqslant x \leqslant 600, y=0 \\
\text { at } 0 \leqslant x \leqslant 600, y=600 \\
\text { at } x=0,0 \leqslant y \leqslant 600 \\
\text { at } x=600,0 \leqslant y \leqslant 600 \\
\text { in } \Omega \text { at } t=0
\end{array}
\end{aligned}
$$

In these equations, $\mathbf{u}, p, t, x$ and $y$ represent the dimensionless velocity, pressure, time and spatial coordinates, respectively. The Reynolds number of the flow is given by $R e=\rho u_{\infty} \ell_{c} / \mu$ and $\Omega_{T}=\Omega \times(0, T)$, with $T$ being the simulation time. The traction boundary condition on the exit section involves the stress tensor $\boldsymbol{\Sigma}$ and uses the notation $a=a^{+}-a^{-}$. It yields a well-posed problem [12] and conveys properly the vortices through the artificial boundary. It requires a reference flow that is selected as the calculated flow on the front of the last column of cells [11]. Finally, $\boldsymbol{\Gamma} \cdot \mathbf{u}$ is the penalization term, which for the DNS, is fixed to be $\boldsymbol{\Gamma}=10^{-16} \mathbf{I}$ so that it vanishes in $\Omega_{f}$. On the opposite, $\boldsymbol{\Gamma}=10^{8} \mathbf{I}$ in $\Omega_{s}$ so that the velocity becomes of the order $10^{-8}$ inside each square. It is worth mentioning that the penalized model in Eqs. (1) is also used to solve the effective-medium equations that are taken to be formally the same as the pore scale model. This represents a heuristic approach since a rigorous derivation of the effective equations is still an active research field. A recent work proposed an up-scaled model that is non-local in time together with the associated closure problems [29]. The up-scaled momentum equation does not correspond to Eq. (1a), but, for the sake of simplicity, the heuristic model of Eqs. (1) is used here as a first approach. For this model, $\mathbf{u}$ and $p$ must be understood as the intrinsic average of the point-wise velocity and pressure. The intrinsic average of any physical quantity $\psi$ is defined by $\langle\psi\rangle^{\beta}=1 / V_{\beta} \int_{\mathrm{V}_{\beta}} \psi d V, \mathrm{~V}_{\beta}$ being the region of the fluid phase (of volume $V_{\beta}$ ) within a periodic unit cell of the porous medium. Consequently, the entire porous medium is conceived as a pseudo-continuum $\left(\Omega_{p}\right)$ as sketched in Fig. 1b. Following the same trend of ideas used for the DNS, $\boldsymbol{\Gamma}=10^{-16}$ in the fluid region, which is now denoted as $\Omega_{\eta}$.

The simplest effective-medium model is obtained by fixing $\boldsymbol{\Gamma}=\frac{\epsilon}{R e} k^{-1} \mathbf{I}$, where $k$ is the dimensionless intrinsic permeability of the porous medium, which is obtained from the solution of an intrinsic closure problem as detailed below. In the following, this model is referred to as the $K$ model. A second-order model, denoted as the $K 2$ model, introduced in [41] following the experiments in [17], is also employed using an Ergun formulation given by $\boldsymbol{\Gamma}=\left(\frac{\epsilon}{R e} k^{-1}+0.1429 \epsilon^{3 / 2} k^{-1 / 2}\|\mathbf{u}\|\right) \mathbf{I}$. In this formulation, $k$ can be estimated from Ergun's formula or from the closure procedure. Both have been tested and, since the two values of $k$ 
differ by more than $50 \%$, they lead to noticeably different flow predictions, especially at large values of the Reynolds number. A better agreement was found with $k$ predicted from a closure scheme and for this reason only this alternative is kept in the analysis in section 4. Finally, a full model involving a more complex form of $\boldsymbol{\Gamma}$ depending on both the local Reynolds number inside the porous medium and the orientation of the local pressure gradient, can be used to improve the approximation [28]. In this model (denoted as the $H$ model) $\boldsymbol{\Gamma}=\frac{\epsilon}{R e} \mathbf{H}^{-1}$, with $\mathbf{H}$ being a full apparent permeability tensor whose components can be predicted by, for example, solving the following ancillary closure problem in a periodic unit cell (such as the one sketched in Fig. 1a)

$$
\begin{gathered}
\nabla \cdot \mathbf{M}=\mathbf{0}, \quad \text { in } \Omega_{f} \\
R e_{\text {local }} \mathbf{u}_{p} \cdot \nabla \mathbf{M}=-\nabla \mathbf{m}+\nabla^{2} \mathbf{M}+\mathbf{I}, \quad \text { in } \Omega_{f} \\
\mathbf{M}=\mathbf{0}, \quad \text { at the solid-fluid interface } \\
\psi\left(\mathbf{r}+\mathbf{l}_{i}\right)=\psi(\mathbf{r}), \quad \psi=\mathbf{M}, \mathbf{m}, i=1,2,3 \\
\langle\mathbf{m}\rangle^{\beta}=\mathbf{0} .
\end{gathered}
$$

Here, $R e_{\text {local }}$ is a local Reynolds number that is defined in terms of $v_{r e f}=\frac{\ell_{c}^{2}}{\mu}\left\|\nabla\langle p\rangle^{\beta}\right\|$. In addition, $\mathbf{u}_{p}=\mathbf{v} / v_{\text {ref }}$, whereas $\mathbf{r}$ and $\mathbf{l}_{i}$ represent a position vector locating points in the fluid phase and each of the lattice vectors of the unit cell, respectively. Once this problem is solved, the apparent permeability tensor is computed as $\mathbf{H}=\epsilon\langle\mathbf{M}\rangle^{\beta}$. In practice a table is built considering the local Reynolds number and the orientation of $\nabla\langle p\rangle^{\beta}$ (see the appendix reporting the values of $\mathbf{H}^{-1}$ ) that is used to compute the local value of $\boldsymbol{\Gamma}$. When $R e_{\text {local }}=0$, the closure problem in Eqs. (2) is intrinsic and the solution on $\mathbf{M}$ provides the intrinsic permeability tensor $\mathbf{K}=\mathbf{H}$. The unit cell employed to generate the porous region here is such that $\mathbf{K}=k \mathbf{I}$. For the value of the porosity used here, the solution of the closure problem yields $k=0.0414$ that is employed in the $K$ model.

\section{Numerical approximation}

The space discretization of the computational domain is based on second and third order finite differences. The time derivative is approximated by a second-order Gear scheme with explicit treatment of the convection terms, which are discretized using a third-order up-flow scheme to insure accuracy [14]. A Courant, Friedrichs and Lewy condition related to the explicit discretization of the convection terms requires a time-step of the same order as the space-step and this is due to the fact that $\mathbf{u}(x=0)=\mathbf{e}_{x}$. All the linear terms are solved implicitly and approximated by second-order centered finite differences.

The whole system in velocity-pressure is solved using a multigrid method. This means that on the square domain $\Omega$, a sequence of uniform grids from the coarsest one, $G 1$ made of $120 \times 120$ cells, to the finest one, G7 including $7680 \times 7680$ cells, are defined by doubling the number of cells in each direction. To have an accurate reference flow with the DNS, a finer grid called $\widetilde{G 8}$ is built with eight levels of grids starting from a grid with $90 \times 90$ cells. This grid contains $9 / 16$ times the number of cells of $G 8$ (i.e., $11520 \times 11520$ cells). Then a multigrid algorithm is applied using a cell-by-cell Gauss-Seidel solver as a smoother. The discretization is performed on staggered grids with the pressure located at the center and the velocity components at the middle of the sides of each grid block in order to enforce the divergence-free condition. Consequently, the use of the smoother implies that the velocity is updated twice and the pressure once at each iteration. This approach is very efficient to capture all the modes of the approximate solution since very few iterations are needed to converge.

Parallelization of the whole program, despite the complexity induced by the multigrid method and the Gauss-Seidel smoother, is carried out using MPI procedures. On the one hand, there are coarse grids on which it is not possible to use a large number of cores. So the number of cores is adapted to the size of the grid as explained in [13]. On the other hand, due to the fact that a Jacobi smoother, that would be much easier to parallelize, does not ensure proper convergence, a Gauss-Seidel smoother was chosen. Consequently, there is a backward dependence that prevents a full parallelization in each direction, thus reducing the efficiency [13]. Nevertheless, a strong scalability of one is obtained on hundreds of cores. In other words the simulations 
performed on 240 cores are running 240 times faster than on a single core.

The penalization term in the $K$ model involves the velocity at the current time with a constant coefficient and does not require any approximation. The penalization term in the $K 2$ model is linearized by taking the norm of the velocity at the previous time step. For the $H$ model, the local components of the tensor $\mathbf{H}$ have to be estimated given the tabulated values versus the Reynolds number and the angle of the local pressure gradient with the main direction of the flow (i.e., the $x$-direction). For the sake of simplicity, the values of the tensor $\mathbf{H}$ are tabulated in multiples of 5 in both the Reynolds number and the angle. More specifically, the values of the components of $\mathbf{H}$ were selected in the table as those corresponding to the nearest values of the Reynolds number and the angle.

The flow inside and around the porous domain is solved for three different Reynolds numbers $R e=10$, $R e=100$ and $R e=1,000$ corresponding to three different regimes as can be seen in Fig. 2. This figure shows snapshots of the vorticity obtained by DNS on $G 7$ for $R e=10$ and on $\widetilde{G 8}$ for the two other Reynolds numbers which require a finer mesh to represent complex unsteady flows. Starting from rest, there is a long transition period to reach the steady regime that is only achieved when the flow has crossed the entire domain, i.e., at about $t=600$. The snapshots reported here are obtained after $t=1,000$. At $R e=10$, steady flow is achieved with a large re-circulation zone and no vortices and almost no flow inside the porous region. At $R e=100$, the flow enters significantly the porous medium at the front and in the vicinity of the traveling vortices, which are large and travel downstream on top of the porous domain from the end of a large re-circulation zone. At $R e=1,000$, the flow is turbulent with many small eddies coming from the front corner. The flow enters the porous medium almost everywhere and near the entrance, the flow is as intense as in the re-circulation zone, as shown in Fig. 3.

To test grid convergence, the flow is computed on the $G 5$ (3,686, 400 cells), $G 6$ (14,745, 600 cells), $G 7(58,982,400$ cells $)$ and $\widetilde{G 8}(132,710,400$ cells $)$ grids. For each case, the velocity components are time averaged over a dimensionless interval of time equal to 3000 so that the flow has traveled several times over the porous medium length. The time average is taken under steady conditions, i.e. for times larger than the time it takes a fluid particle to travel through the whole domain. For a detailed qualitative comparison of the solutions, the mean velocity profiles transverse to the flow are compared. These profiles are represented at three different horizontal positions, namely $x=200$, corresponding approximately to the middle of the re-circulation zone, $x=400$, that corresponds to $3 / 4$ of the porous region on top of which the vortices are traveling and $x=500$, corresponding to the exit section of the porous medium. In Fig. 4 are plotted the mean profiles of the two velocity components at $R e=100$ for the reference flow obtained by DNS on the $\widetilde{G 8}$ grid and by the $K$ model on the $G 6$ grid. The two components show about the same discrepancies, however the horizontal component $u$ reveals more features in the porous part and on top of the domain. So in the following only the u-component profiles are presented.

The grid convergence of the DNS is achieved comparing the solutions obtained on the four grids mentioned above. At $R e=10$, the velocity profiles on the $G 7$ and $G 8$ grids are superimposed as the flow barely enters the porous domain. Thus the reference flow in the following corresponds to the $G 7$ computation. For higher Reynolds number values it is necessary to use the $\widetilde{G 8}$ grid to properly represent the flow inside a porous unit-cell. At $R e=100$, Fig. 5 and table 1 show that, at least, a $G 6$ grid is necessary to achieve a relative error below $5 \%$, taking the reference velocity obtained on the $\widetilde{G 8}$ grid. The velocity profiles computed on the $G 7$ and $\widetilde{G 8}$ grids are not fully superimposed as shown on the zooms represented in Fig. 5. However the relative difference is around $1 \%$ on average, with a maximum of $1.6 \%$ in the middle of the domain. Consequently, in the next sections, the reference flow at $R e=100$ and $R e=1,000$ refers to the DNS solution computed on the $\widetilde{G 8}$ grid.

Table 1: Percentage of Euclidian relative error of the velocity profiles at $R e=100$ with respect to the reference flow taken as the DNS results on the $\widetilde{G 8}$ grid.

\begin{tabular}{|c||c|c|c|}
\hline & G5 & G6 & G7 \\
\hline \hline Profile $x=200$ & $3.44 \%$ & $0.68 \%$ & $0.48 \%$ \\
\hline Profile $x=400$ & $11.72 \%$ & $3.2 \%$ & $1.6 \%$ \\
\hline Profile $x=500$ & $8.99 \%$ & $1.98 \%$ & $0.76 \%$ \\
\hline
\end{tabular}



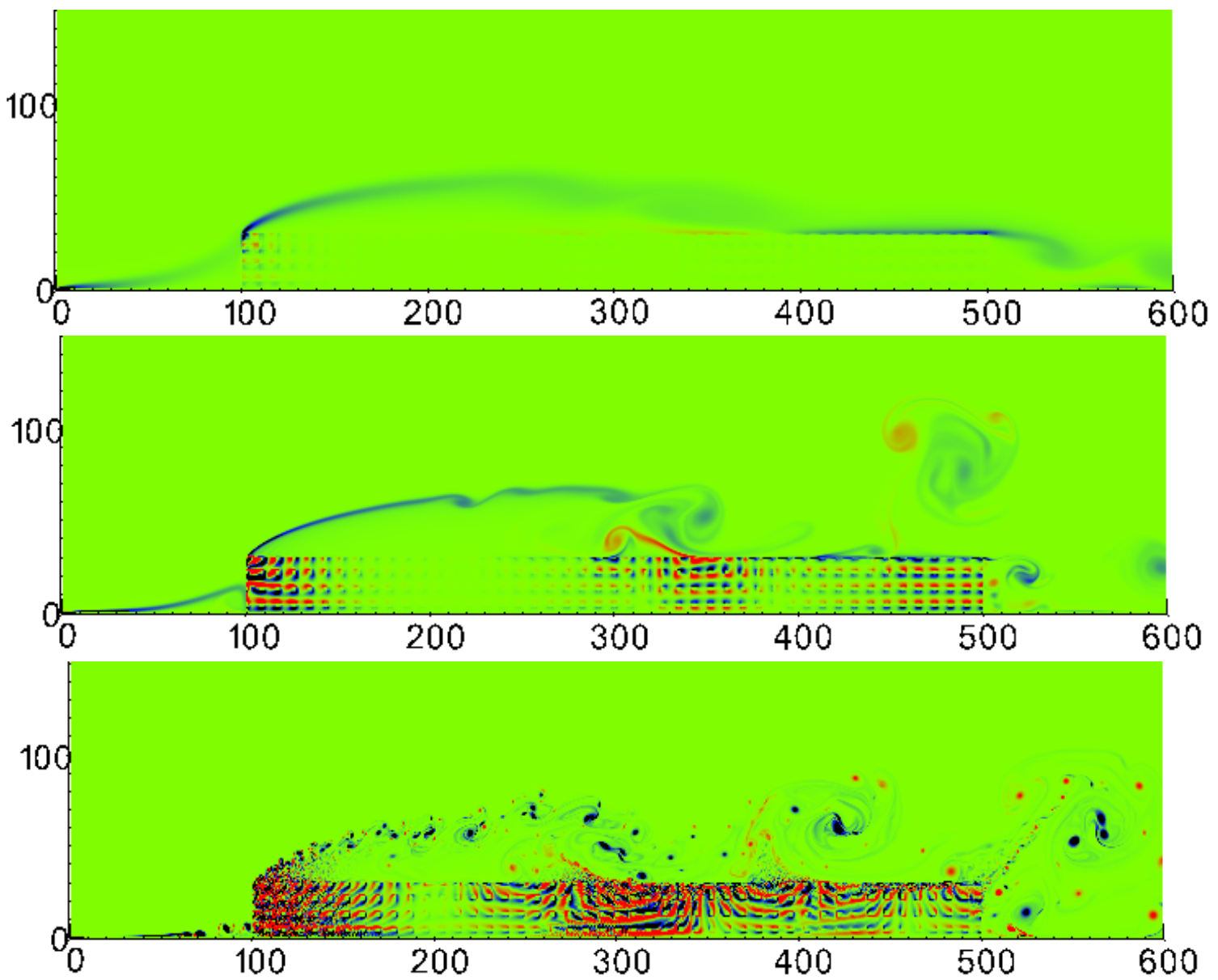

Figure 2: Instantaneous vorticity fields at $R e=10$ (top), $R e=100$ (middle) and $R e=1,000$ (bottom) in the bottom of the domain. To better compare the various regimes, the vorticity ranges from -0.5 (dark blue) to 0.5 (dark red). The green colour corresponds to zero vorticity.

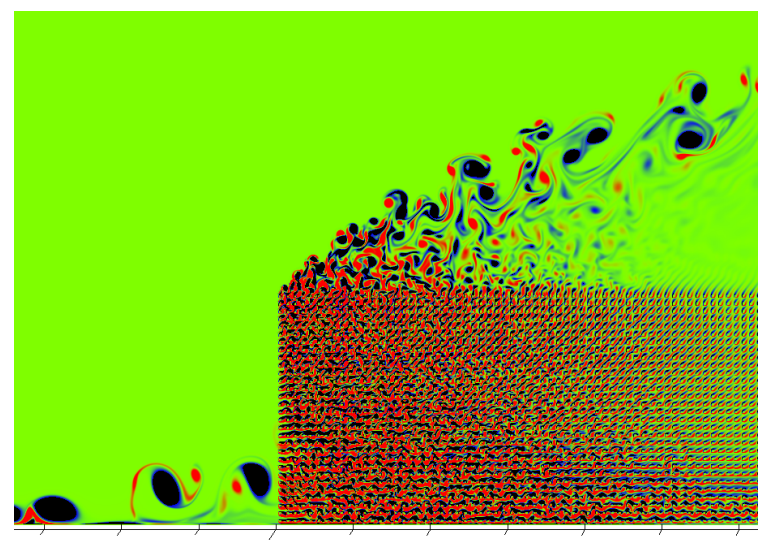

100

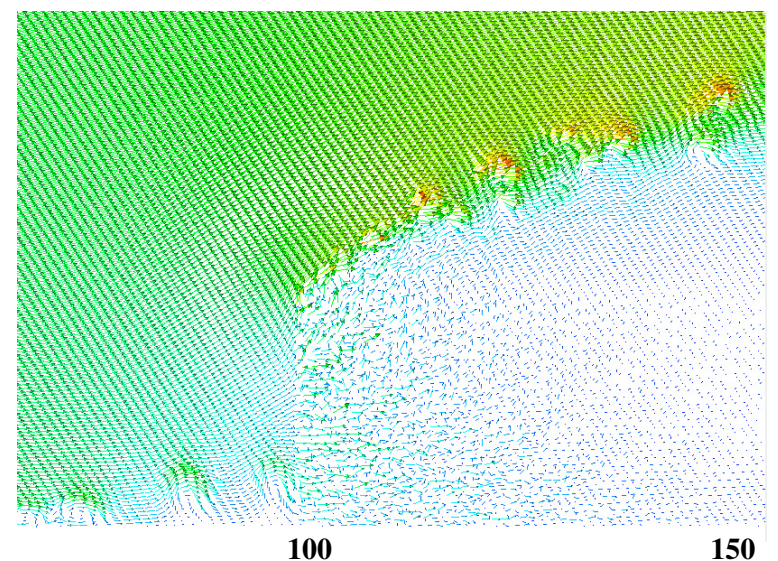

100
150

Figure 3: Zoom of the instantaneous vorticity field (left) and of the velocity field (right) around the front of the porous domain at $R e=1,000$. The vorticity ranges from -0.5 (dark blue) to 0.5 (dark red). The length and color of the velocity vectors are representative of the velocity magnitude from 0 (dark blue) to the maximum (dark red). 

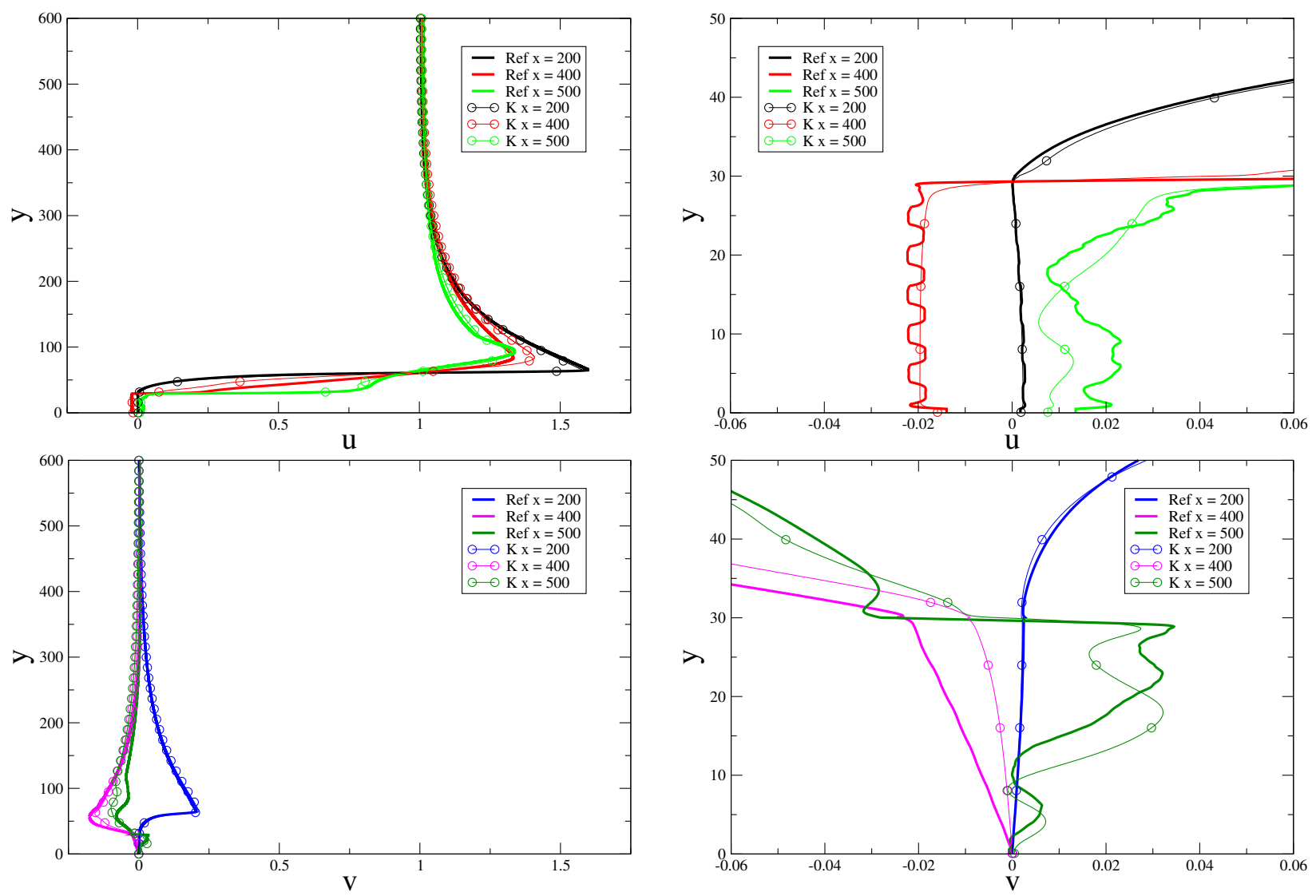

Figure 4: Profiles of the horizontal ( $\mathrm{u}$ for the top row) and vertical ( $\mathrm{v}$ for the bottom row) mean velocity components at three different vertical sections positioned along the $x$-direction at 200, 400 and 500 for $R e=100$ computed with the $K$ model on the $G 6$ grid and from DNS on the $\widetilde{G 8}$ grid. Left: in the whole domain. Right: inside and above the porous region.

Regarding the convergence of the effective-medium models, it depends on the Reynolds number and more precisely on the flow regime. For the steady flow at $R e=10$, the convergence is achieved for the three models on the $G 6$ grid as well as for the unsteady flow at $R e=100$. The behaviour is slightly different for the turbulent flow at $R e=1,000$. In Fig. 6 the profiles in the middle part for $K$ and $H$ models are plotted on the two consecutive grids $G 6$ and $G 7$. For the $K$ model, the profiles are very close and it shall be considered that the convergence is still obtained on the $G 6$ grid. However, for the $H$ model there are more discrepancies. This is not surprising as for this model, at high Reynolds number, the coefficients of the tensor $\mathbf{H}$ are not constant but depend strongly on the local flow. It should be mentioned that the maximum relative percent difference between the results obtained with the $G 6$ and $G 7$ grids is below $5 \%$ and this can be considered acceptable for mesh convergence. A further remeshing would lead to a simulation as costly as the DNS and it is not carried out here.

To make a fair comparison, it is important to take into account the CPU time to compute the solution at a given time. Here are compared the CPU times at $R e=100$ taking the time for DNS on the finest $\widetilde{G 8}$ grid as the reference time. The percentage of time of each computation with respect to the reference time is given in table 2. It is clear that there is a tremendous time saving using the penalization models on coarser grids. Even the full model on the $G 7$ grid gives a significant reduction of time. Moreover these models allow using a coarser grid to obtain a solution close to the DNS solution on the finest grid. According to the above convergence analysis, results obtained with the effective-medium models are mainly reported for the $G 6$ grid and also for the $G 7$ grid for the $H$ model at high Reynolds numbers. 

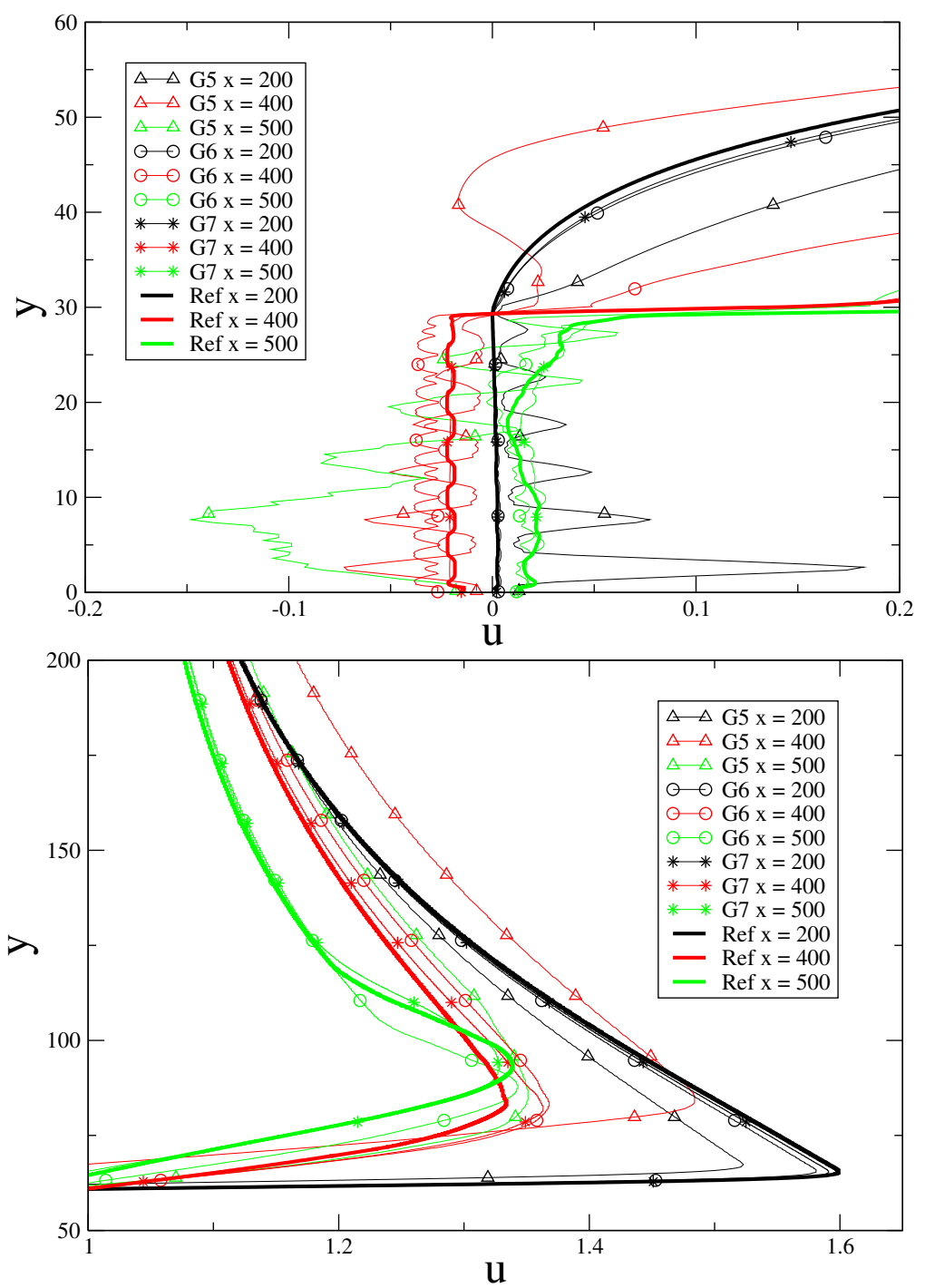

Figure 5: Convergence of the mean u-velocity profiles at three different vertical sections positioned along the $x$-direction at 200, 400 and 500 with respect to the grid size $G 5, G 6, G 7$ and $\widetilde{G 8}$ for $R e=100$. Top: inside and above the porous rectangle, bottom: in the middle part above the porous rectangle.

Table 2: Percentage of time to achieve the solution at a given time for various methods and grids at $R e=100$. The reference time is obtained for the DNS on the finest $\widetilde{G 8}$ grid. All the computations are performed on 240 cores.

\begin{tabular}{|c||c|c|c|c|c|}
\hline Method and grid & DNS G8 & K G6 & K2 G6 & H G6 & H G7 \\
\hline \hline Percentage of time & $100 \%$ & $2 \%$ & $2.3 \%$ & $3.5 \%$ & $27 \%$ \\
\hline
\end{tabular}

\section{Numerical results}

In this section results obtained with the effective-medium model are compared to those from DNS for three different flow regimes in order to investigate the influence of the Reynolds number. The profiles of the horizontal velocity component, $\mathrm{u}$, are represented for three vertical sections of the domain (close to the vertical fluid-porous medium interface, $x=200$, at the center of the porous domain, $x=400$, and near 

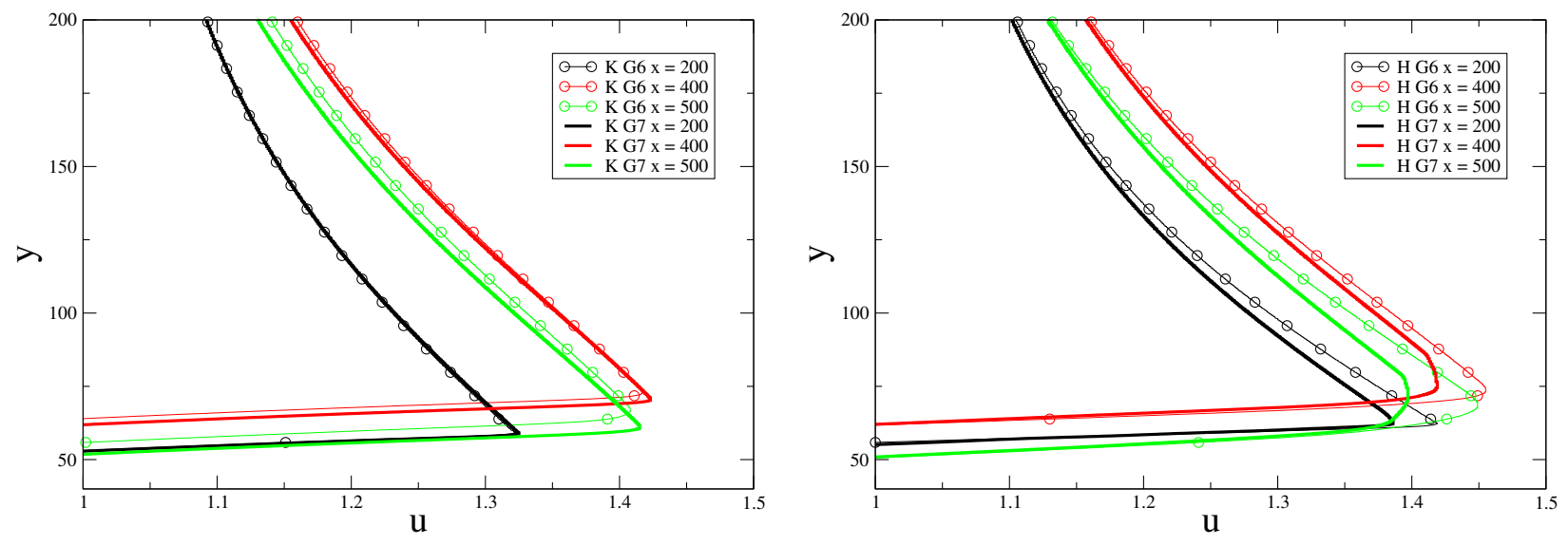

Figure 6: Mean u-velocity profiles at three different vertical sections positioned along the $x$-direction at 200, 400 and 500 with respect to the grids $G 6$ and $G 7$ in the middle part above the porous rectangle for $R e=1,000$. Left: the $K$ model, right: the $H$ model.

the exit of the porous region, $x=500$ ). This is done in the whole domain and zooms are provided inside the porous domain, above it where the velocity reaches a maximum, and at the top for the far-field. For comparison, DNS results are averaged over a domain corresponding to a geometrical unit cell of the porous region. The center of this domain is at the $x$-coordinate of the section of interest while it is moved along the $y$-direction in order to reconstruct the profile of the average velocity.

At $R e=10$, all the effective medium $K, K 2$ and $H$ models computed on the $G 6$ grid yield the same profiles that are globally in good agreement with the DNS predictions in the entire domain as can be seen in Fig. 7. The flow is almost zero everywhere in the porous region like for DNS. However, there are some noticeable differences at the maximum velocity, particularly at $x=400$, which corresponds to the re-attachment of the recirculation zone. For this low Reynolds number the full apparent permeability tensor almost reduces to $\mathbf{H}=k \mathbf{I}$ and, in the $K 2$ model, the second term in $\boldsymbol{\Gamma}$ remains negligible with respect to the first one explaining why the three effective models coincide. Therefore the accuracy achieved by the three models is the accuracy of the $K$ model because the corrections do not apply in this case.

For $R e=100$, the solutions obtained with the three models computed on the $G 6$ grid are compared in Fig. 8 to the reference solution computed by DNS on the $\widetilde{G 8}$ grid. The whole profile is in good agreement with the reference one. However the zooms in fig. 8 show that there are significant differences between the three models because, for this Reynolds number value, the corrections are not negligible. Indeed, although the profiles are very similar overall, the behaviour of the $K 2$ model at $x=200$ is very different above the porous rectangle, thus revealing a poor position of the re-circulation zone. In the middle part of the domain, the $H$ model performs much better than the other two as indicated by the profile that is almost superimposed on the reference profile.

For the Reynolds number values up to 100 investigated so far, for which the flow remains laminar, the penalization models, especially the $H$ model, are able to represent properly the flow inside and around a porous obstacle. Hence, it is now of interest to examine the performance of the models when increasing the Reynolds number value to reach a turbulent regime as shown in Fig. 2 for $R e=1,000$. Even if the penalization models are built to compute the flow inside a porous medium, it remains that the main difficulty is related to the high speed flow in front of the porous domain. The full model is the only one able to take into account the characteristics of the flow, namely the local Reynolds number and the pressure gradient, inside the porous region. To better understand this situation, it is of interest to compare only the $K$ model and the $H$ model to the DNS computed on the finest $\widetilde{G 8}$ grid. Taking into account the convergence study, the $K$ model is simulated on the $G 6$ grid and the $H$ model on the $G 7$ grid. Figure 9 shows that the results compared to the reference flow are very poor for both models. This is particularly significant in the porous domain at $x=200$ where the normal flow enters too much inside the porous rectangle with a velocity one order of magnitude larger than expected. At this location, the velocity inside the porous domain is twice larger with the $K$ model than with the $H$ model. This is probably due to the fact that the tensor is adapted 

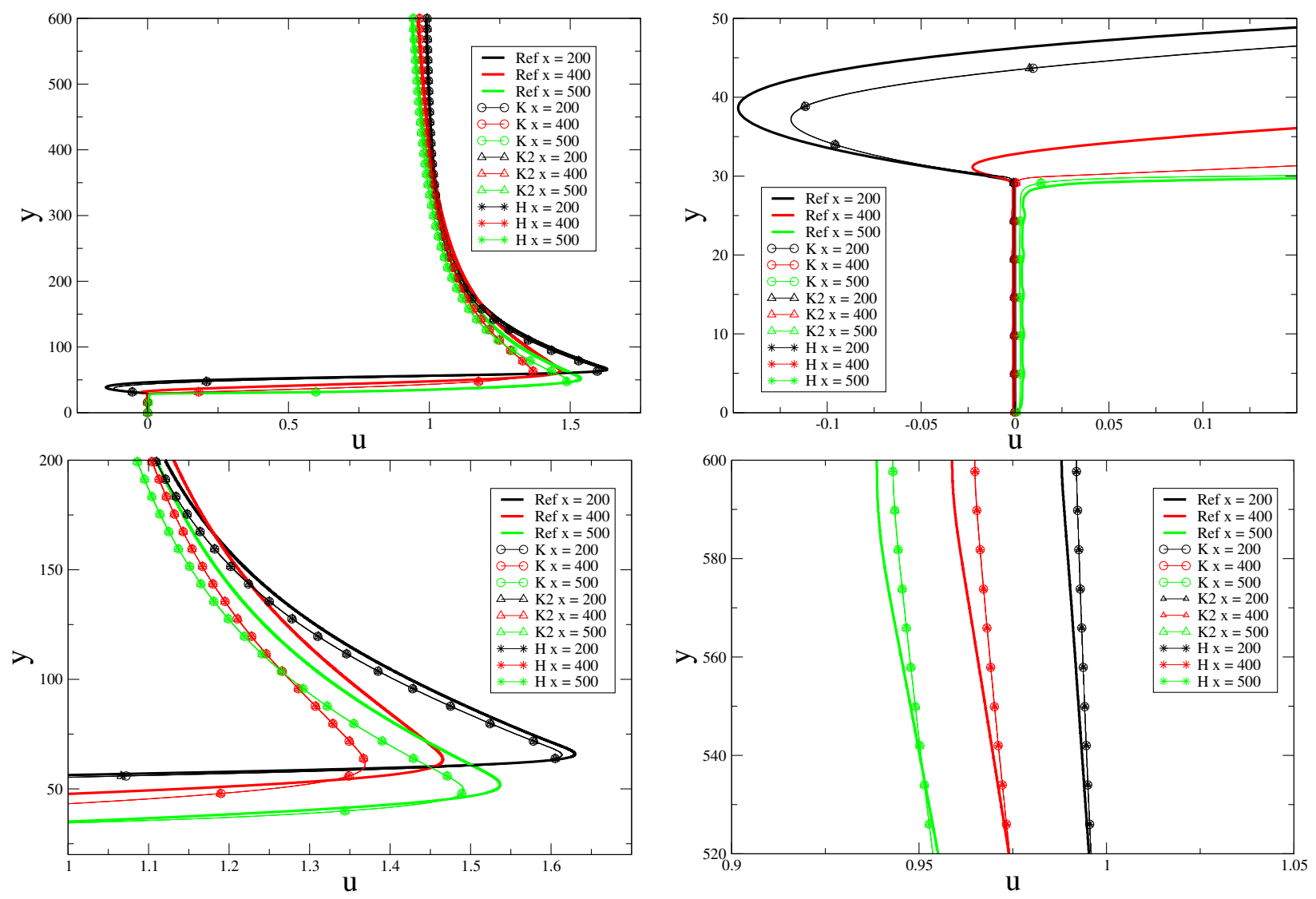

Figure 7: Mean velocity profiles at three different vertical sections positioned along the $x$-direction at 200, 400 and 500 for $R e=10$. Top left: in the whole domain. Top right: inside and above the porous region. Bottom left: in the middle part. Bottom right: at the top of the domain. The three penalization models computed on the $G 6$ grid are compared to the reference flow computed by DNS on the $G 7$ grid.

to the flow with the $H$ model. At other locations the two models give about the same result, which is much closer to the reference. In the middle of the domain, as well as on top of it, both models give a solution very far from the reference one. For this regime, it must be noted that the penalization models give a fairly poor approximation of the actual flow. This is an indication that the limit of the use of the penalization models is reached as they are not built for such flow conditions.

With the aim of exploring the possibility to improve the predictive capabilities of the effective-medium penalized model, an alternative approach is presented in Appendix B in which the values of $\boldsymbol{\Gamma}^{-1}$ are decreased locally near the porous medium boundaries. This is done by replacing $\boldsymbol{\Gamma}^{-1}$ taking into account changes in the horizontal position by postulating $\tilde{\boldsymbol{\Gamma}}^{-1}=4 \boldsymbol{\Gamma}^{-1} /(144-x)$ for $100 \leq x \leq 140$ and the resulting model is denoted as the $K-K$ model. Another alternative is to propose a modification of $\boldsymbol{\Gamma}^{-1}$ taking into account the local changes of the velocity, i.e., $\tilde{\boldsymbol{\Gamma}}^{-1}=\boldsymbol{\Gamma}^{-1} /[100(\|\mathbf{u}\|-0.1)]$ when $\|\mathbf{u}\|>0.11$ and the resulting model is referred to as the $K-U$ model. The numerical simulations show that the $K-K$ and $K-U$ models provide the same performance near the porous medium entrance; however downstream, the $K-U$ model yields better predictions than the $K-K$ model. Finally, when the predictions from the $K-U$ model are compared with those of the $H$-model, improvements are only found above the porous medium.

\section{Conclusions}

In this work, the performance of an effective-medium model for a fluid-porous medium system was investigated by means of comparisons with reference DNS of the pore-scale model under different flow regimes. 

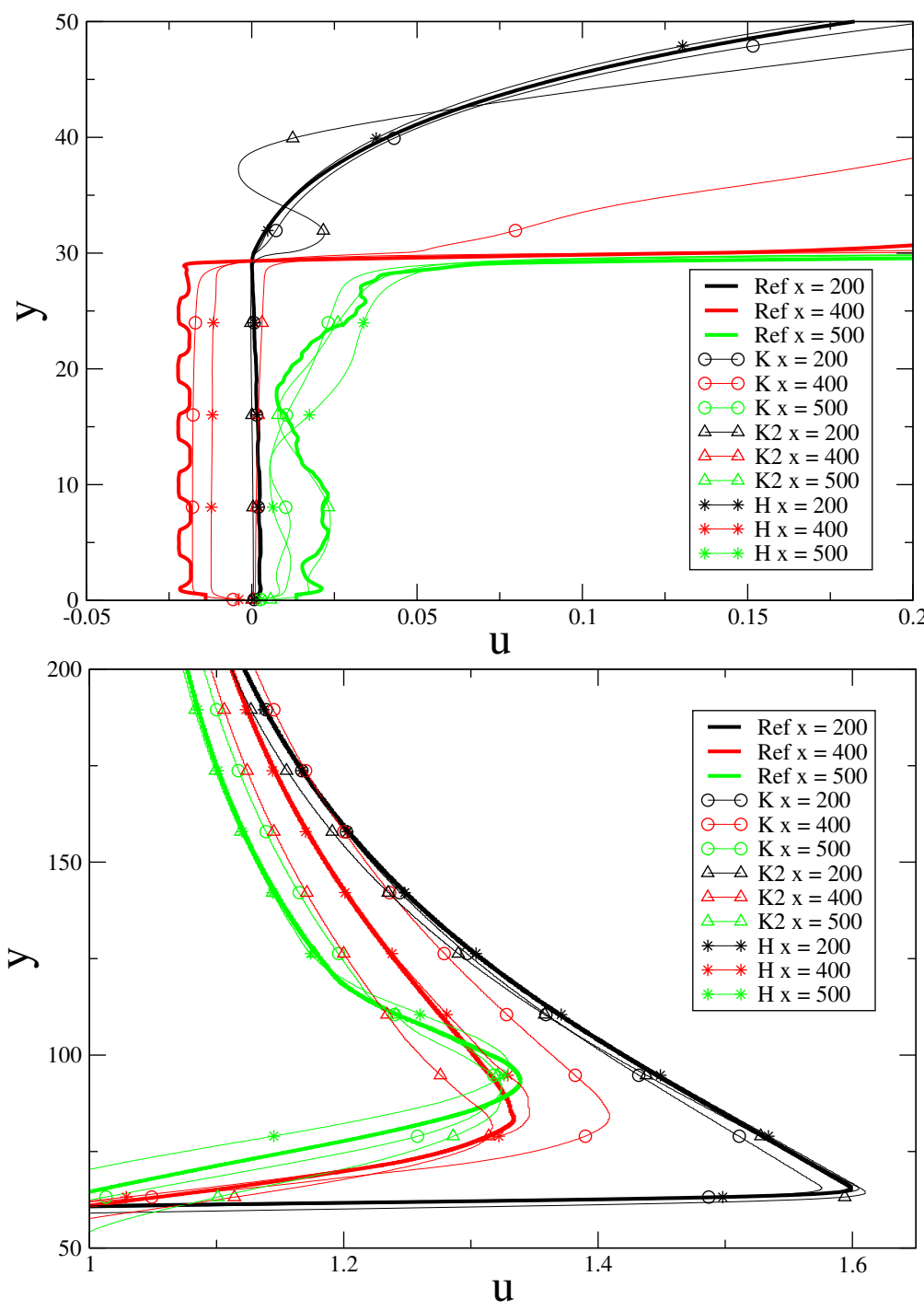

Figure 8: Mean velocity profiles at three different vertical sections positioned along the $x$-direction at 200, 400 and 500 for $R e=100$. Top: inside and above the porous rectangle, bottom: in the middle part above the porous rectangle. The three penalization models computed on the $G 6$ grid are compared to the reference flow computed by DNS on the G8 grid.

The system under consideration is more general than the classical BJ configuration and the DNS results may certainly serve as a reference for subsequent studies on the subject ${ }^{1}$. At both scales, a penalization method was employed to carry out the numerical solution using the same penalized Navier-Stokes equations solver. The penalization term in the effective-medium model was estimated by using the predictions from a closure problem solution that was derived in the homogeneous part of the porous medium. The advantage of this effective-medium approach lies in the fact that promising predictions can be obtained at a significantly less expensive computational cost than the DNS.

It was found that, at sufficiently small Reynolds number values $(R e \simeq 10)$, all the estimations from the penalization term in the effective medium model yield the same results that are not in full agreement with the reference solution. At intermediate Reynolds number values $(R e \simeq 100)$, the effective medium model involving a penalization coefficient that takes into account the full tensorial nature of the apparent permeability (i.e., the $H$-model) was shown to give excellent results on a coarse grid which are very close to

${ }^{1}$ DNS results are available from the authors upon request. 

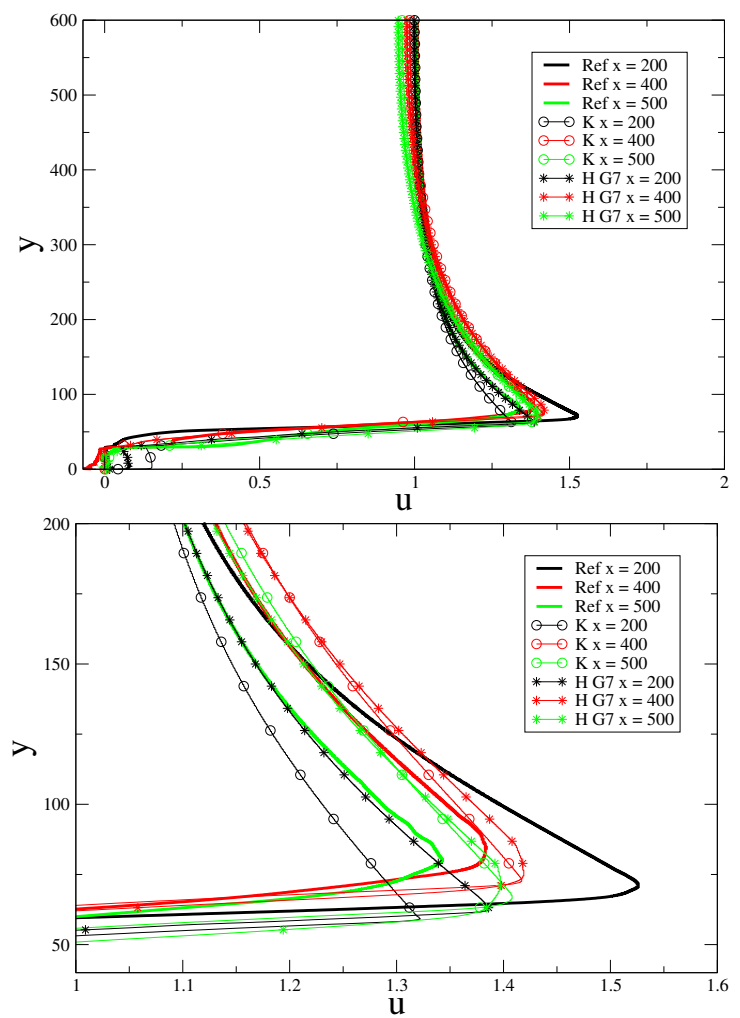
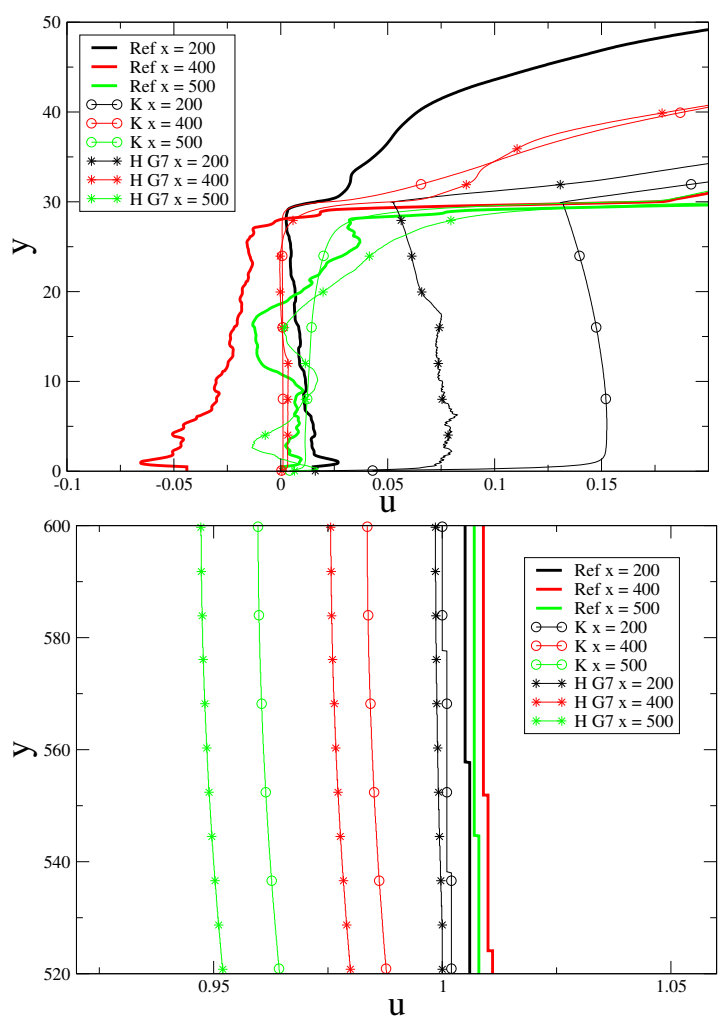

Figure 9: Mean velocity profiles at three different vertical sections positioned along the $x$-direction at 200, 400 and 500 for $R e=1,000$. Top left: the whole domain, top right: inside and above the porous rectangle, bottom left: in the middle part and bottom right: on top of the domain. The $K$ model computed on the $G 6$ grid and the $H$ model computed on the $G 7$ grid are compared to the reference flow computed by DNS on the $\widetilde{G 8}$ grid.

those obtained by DNS on the fine grid. This translates in a drastic computational time reduction of about 30 times. At high Reynolds number for turbulent flows $(R e \simeq 1,000)$, the effective medium models were shown to compare poorly to the reference. In particular, significant discrepancies were observed near the macroscopic boundaries of the porous medium with the fluid region. With the intention of improving the predictive capability of the effective medium model, two heuristic modifications were proposed on the effective model operating in the creeping regime, i.e., the $K$ model. It was found that a modification considering the norm of the local velocity improved the performance at specific positions of the system.

As a matter of prospective, results obtained here should encourage further modeling efforts to derive accurate effective models that are applicable near the fluid-porous medium interface in the turbulent regime. This could be accomplished by formally deriving and solving a closure scheme that takes into account the variations of both the structure and the flow in the interfacial region. In particular, it is of interest to investigate the possibility of predicting the spatial variations of the penalization coefficient $\boldsymbol{\Gamma}^{-1}$ near the boundaries as a function of the flow conditions for any pore topology.

\section{Appendix A}

The values of the components of the tensor $\mathbf{H}^{-1}$ are computed for a square pattern of parallel cylinders of square cross section having a porosity $\epsilon=0.913$ and a flow orthogonal to the cylinders axes. The components 
$H_{x x}, H_{y x}, H_{x y}$ and $H_{y y}$, of tensor $\mathbf{H}$ are satisfying the following properties:

$$
\begin{aligned}
& H_{x x}\left(\theta, R e_{\text {local }}\right)=H_{y y}\left(90^{\circ}-\theta, R e_{\text {local }}\right), \quad H_{y x}\left(\theta, \operatorname{Re}_{\text {local }}\right)=H_{x y}\left(90^{\circ}-\theta, \operatorname{Re}_{\text {local }}\right) \\
& H_{x y}\left(\theta, \operatorname{Re}_{\text {local }}\right)=H_{y x}\left(90^{\circ}-\theta, \operatorname{Re}_{\text {local }}\right), \quad H_{y y}\left(\theta, \operatorname{Re}_{\text {local }}\right)=H_{x x}\left(90^{\circ}-\theta, \operatorname{Re}_{\text {local }}\right) .
\end{aligned}
$$

Then, for $\theta$ from $95^{\circ}$ to $180^{\circ}$ the components of the tensor are given by:

$$
\begin{gathered}
H_{x x}\left(\theta, R e_{\text {local }}\right)=H_{x x}\left(180^{\circ}-\theta, \operatorname{Re}_{\text {local }}\right), \quad H_{y x}\left(\theta, \operatorname{Re}_{\text {local }}\right)=-H_{y x}\left(180^{\circ}-\theta, \operatorname{Re}_{\text {local }}\right) \\
H_{x y}\left(\theta, \operatorname{Re}_{\text {local }}\right)=-H_{x y}\left(180^{\circ}-\theta, \operatorname{Re}_{\text {local }}\right), \quad H_{y y}\left(\theta, \operatorname{Re}_{\text {local }}\right)=H_{y y}\left(180^{\circ}-\theta, \operatorname{Re}_{\text {local }}\right) .
\end{gathered}
$$

Finally, for $\theta$ from $185^{\circ}$ to $360^{\circ}$ the components of the tensor are given by:

$$
\begin{gathered}
H_{x x}\left(\theta, \operatorname{Re}_{\text {local }}\right)=H_{x x}\left(360^{\circ}-\theta, \operatorname{Re}_{\text {local }}\right), \quad H_{y x}\left(\theta, \operatorname{Re}_{\text {local }}\right)=-H_{y x}\left(360^{\circ}-\theta, \operatorname{Re}_{\text {local }}\right) \\
H_{x y}\left(\theta, \operatorname{Re}_{\text {local }}\right)=-H_{x y}\left(360^{\circ}-\theta, \operatorname{Re}_{\text {local }}\right), \quad H_{y y}\left(\theta, \operatorname{Re}_{\text {local }}\right)=H_{y y}\left(360^{\circ}-\theta, \operatorname{Re}_{\text {local }}\right) .
\end{gathered}
$$

The same symmetries are applicable to the tensor $\mathbf{H}^{-1}$, the component values of which are provided as additional material.

\section{Appendix B}

In this appendix, a heuristic modification of the $K$ model is explored. The idea is to investigate the possibility of keeping the same form of the effective-medium momentum equation, which has the advantage of being rather simple and solved with the same numerical code, by empirically modifying the Darcy-like penalization term.

An examination of the flow around the front of the porous region with the $K$ model reveals that there is almost no transition between the fluid and the porous medium (see Fig. B2 left) at $R e=1,000$. The zoom of the velocity field corresponds to the same part of the domain shown in Fig. 3. Indeed, for the $K$ model the mean velocity inside the porous domain (averaged with respect to y, which varies from 0 to 30 ) near the porous medium entrance is quite large. It ranges from 0.83 at $x=100$ to 0.14 at $x=200$ (Table B1) instead of 0.33 at $x=100$ and 0.01 at $x=200$ for the reference flow. Data reported in Table B1 show that due to the re-circulation zone on top of the porous medium, the mean velocity inside the porous region becomes negative in the second part of this re-circulation zone, namely between $x=250$ and $x=300$. However, the velocity is always positive with the $K$ model. In this model, the coefficient $k / \epsilon$ in $\boldsymbol{\Gamma}$ is equal to 0.0453 in the

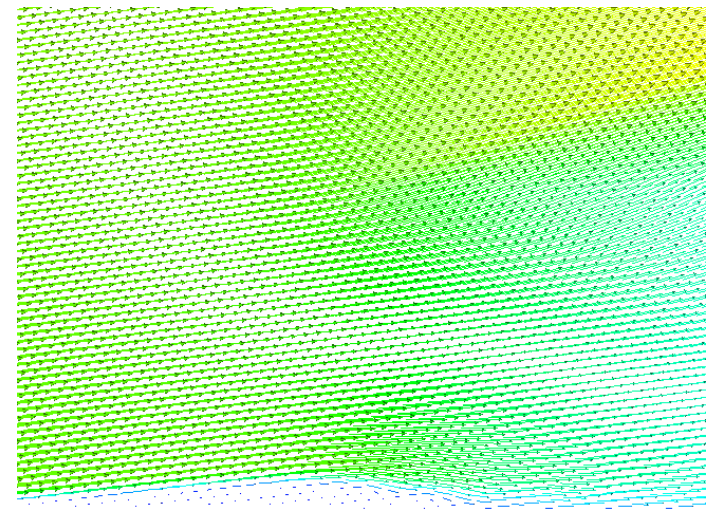

100

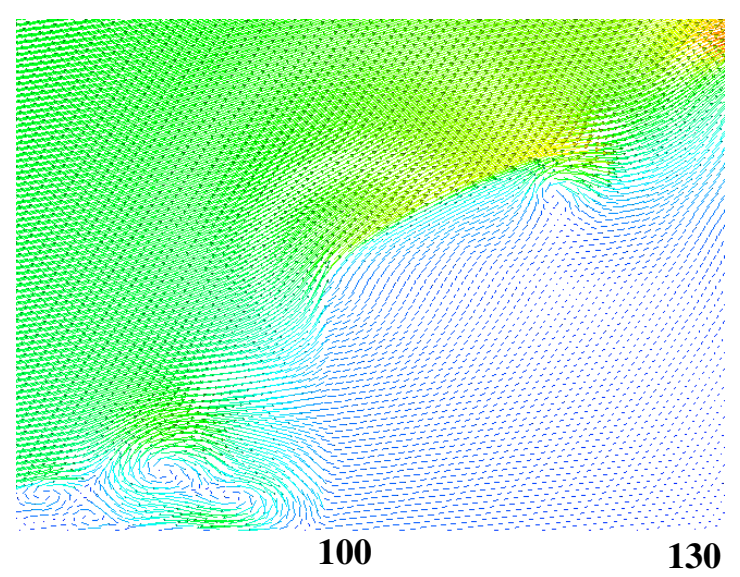

Figure B2: Zoom at the same location of the instantaneous velocity field around the front of the porous rectangle for $R e=1,000$. Left: the $K$ model, right: the improved $K-U$ model. Both models are computed on the $G 6$ grid. The length and color of the velocity vectors are related to the velocity magnitude from 0 (dark blue) to the maximum (dark red).

present computation. For $R e=1,000$, it yields $\boldsymbol{\Gamma}^{-1}=45.3 \mathbf{I}$. This value seems correct in the middle part of 
the porous medium but does not perform well near the fluid-porous medium boundary, specially when there is a strong normal velocity ahead. To decrease the velocity inside the porous medium close to the surface, it is necessary to decrease locally the value of $\boldsymbol{\Gamma}^{-1}$. This can be done, for example, by arbitrarily dividing $\boldsymbol{\Gamma}^{-1}$ by an order of magnitude at the boundary and then increasing it linearly in the first $10 \%$ of the rectangle size to reach the right value $\boldsymbol{\Gamma}^{-1}=45.3 \mathbf{I}$ and keeping it until the exit section of the porous medium. This can be done by replacing $\boldsymbol{\Gamma}^{-1}$ by $\tilde{\boldsymbol{\Gamma}}^{-1}$ defined as $\tilde{\boldsymbol{\Gamma}}^{-1}=\frac{4}{144-x} \boldsymbol{\Gamma}^{-1}$ for $100 \leq x \leq 140$. The resulting model is called the $K-K$ model in the following.

Table B1: Comparison of the mean velocity inside the porous rectangle between the reference flow computed by DNS on the $\widetilde{G 8}$ grid and the three $K$ models computed on the $G 6$ grid at various locations. The mean velocity is averaged with respect to $\mathrm{y}$ which varies from 0 to 30 .

\begin{tabular}{|c||c|c|c|c|c|}
\hline Location & $\mathrm{x}=100$ & $\mathrm{x}=150$ & $\mathrm{x}=200$ & $\mathrm{x}=250$ & $\mathrm{x}=300$ \\
\hline \hline DNS on $\widetilde{G 8}$ & 0.33 & 0.07 & 0.01 & -0.006 & -0.03 \\
\hline$K$ model on $G 6$ & 0.83 & 0.37 & 0.14 & 0.045 & 0.008 \\
\hline$K-K$ model on $G 6$ & 0.3 & 0.07 & 0.008 & -0.018 & -0.042 \\
\hline$K-U$ model on $G 6$ & 0.27 & 0.06 & -0.018 & -0.035 & -0.032 \\
\hline
\end{tabular}

The solution computed with the $K-K$ model has a mean value very close to the reference flow in the entrance region of the porous medium, mostly for $100 \leq x \leq 200$. This is very encouraging but the flow computed with the $K-K$ model is still quite different from the reference flow further into the porous medium (see Fig. B3). An alternative way to modify the penalization coefficient for the effective medium $K$ model is to relate this coefficient to the local velocity magnitude. Then the penalization coefficient can be modulated not only in the front part of the porous medium but within the whole porous medium. With this approach it is possible, in principle, to also encompass the situation when a strong vortex travels down on top of the porous medium. When the velocity magnitude exceeds a chosen threshold, the penalization coefficient is reduced accordingly. Analyzing the velocity inside the porous medium, this can be applied when the velocity magnitude exceeds a value around 0.1. Therefore, a modified value of $\boldsymbol{\Gamma}$ can be proposed as $\tilde{\boldsymbol{\Gamma}}^{-1}=\frac{1}{100(\|\mathbf{u}\|-0.1)} \boldsymbol{\Gamma}^{-1}$ if $\|\mathbf{u}\|>0.11$. The resulting model is called the $K-U$ model in the following. As
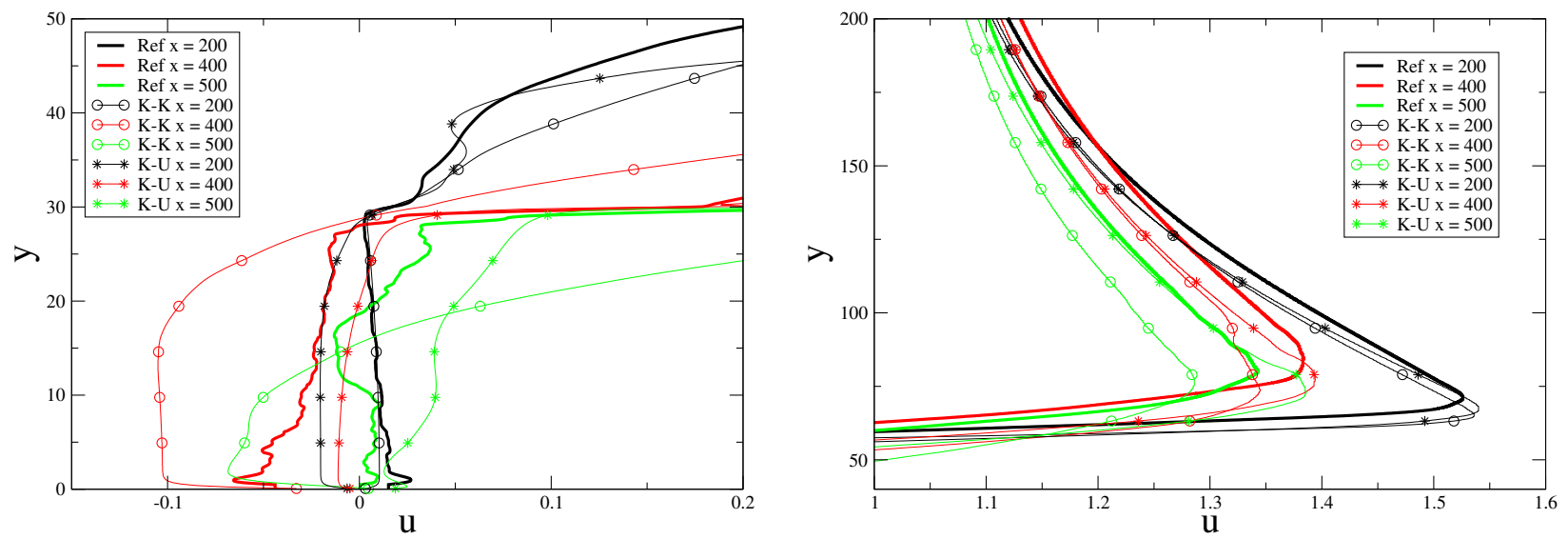

Figure B3: Mean velocity profiles at three different vertical sections positioned along the $x$-direction at 200, 400 and 500 for $R e=1,000$. Left: zoom around the front of the porous rectangle, right: zoom in the middle part above the porous rectangle. The two penalization models computed on the G6 grid are compared to the reference flow computed by DNS on the $\widetilde{G 8}$ grid.

shown in Table B1, the $K-U$ model does not perform better than the $K-K$ model in the front part of the porous medium. However, the predictions are much improved downstream as the $K-K$ model yields a very poor approximation (Fig. B3 left). Moreover, the approximation is much better with the $K-U$ model in 
the whole domain at the exit section (Fig. B3 right). The empirical improvements made on the $K$ model, make possible to find a coherent behaviour at the front of the porous body as shown in Fig. B2 right. The porous rectangle is now clearly visible as well as the re-circulation above like in Fig. 3 right for the DNS. As expected the two snapshots can not be directly superimpose because they represent instantaneous solutions that are necessarily different with two approaches so far apart which have a different transient. In addition the Kelvin-Helmholtz vortices are smaller on the finest $\widetilde{G 8}$ grid. However, what is captured by the effective models is the mean flow behaviour.
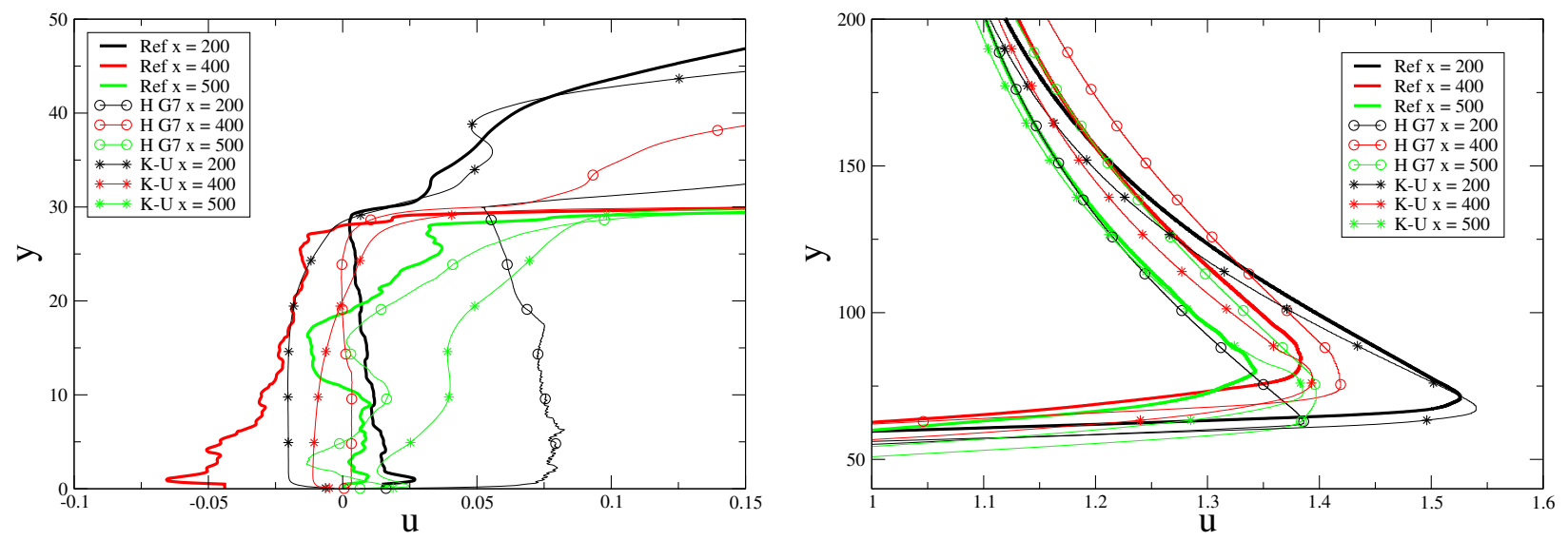

Figure B4: Mean velocity profiles at three different vertical sections positioned along the $x$-direction at 200, 400 and 500 for $R e=1,000$. Left: zoom around the front of the porous rectangle, right: zoom in the middle part above the porous rectangle. The $K-U$ model computed on the $G 6$ grid is compared to the reference flow computed by DNS on the $\widetilde{G 8}$ grid and to the $H$ model computed on the $G 7$ grid.

To conclude this section, it is worth comparing the performance of the $K-U$ model computed on the $G 6$ grid and the best one obtained so far, i.e., the $H$ model computed on the $G 7$ grid, with respect to the DNS reference obtained on the $\widetilde{G 8}$ grid for $R e=1,000$. In Fig. B4, the corresponding horizontal velocity profiles, $\mathrm{u}$, are presented. In the porous region (left graph of Fig. B4), the modification on $\boldsymbol{\Gamma}$ in the $K-U$ model improves significantly the prediction of $u$ at the entrance, although a re-circulation is obtained which does not correspond to the reference flow. The improvement is less significant at the center of the porous region whereas the prediction deteriorates at the exit. Above the porous region, (right part of Fig. B4), the $K-U$ model improves the prediction of $\mathrm{u}$ in all the three investigated sections, in particular at $x=200$. The effective integration of the interface, in the case of high speed, to compute the full tensor $\mathbf{H}$ should provide results close to the DNS. Finally, it must be pointed out that the $K-U$ model only requires $2 \%$ of the DNS CPU time.

\section{Acknowledgments}

All the numerical simulations have been run on PLAFRIM platform supported by IMB, University of Bordeaux, and INRIA Bordeaux-Sud Ouest.

\section{Compliance with ethical standards}

\section{Funding}

There is no funding for this work. 


\section{Conflict of interest}

The authors declare that they have no conflict of interest.

\section{References}

[1] S.R. Ahmed, G. Ramm, and G. Faltin. Some salient features of the time-averaged ground vehicle wake. In SAE Technical Paper. SAE International, 02 1984. doi: 10.4271/840300. URL https://doi.org/10.4271/840300.

[2] Ph. Angot, C.H. Bruneau, and P. Fabrie. A penalization method to take into account obstacles in incompressible viscous flows. Numerische Mathematik, 81(4):497-520, 1999.

[3] M. N. Ardekani, L. Al Asmar, F. Picano, and L. Brandt. Numerical study of heat transfer in laminar and turbulent pipe flow with finite-size spherical particles. International Journal of Heat and Fluid Flow, 71:189-199, jun 2018. doi: 10.1016/j.ijheatfluidflow.2018.04.002.

[4] E. Arquis and J. P. Caltagirone. Sur les conditions hydrodynamiques au voisinage d'une interface milieu fluide-milieux poreux: application à la convection naturelle. Comptes Rendus de l'Academie des Sciences, 299:1-4, 1984.

[5] J.-L. Auriault. On the domain of validity of Brinkman's equation. Transport in Porous Media, 79(2): 215-223, dec 2009. doi: 10.1007/s11242-008-9308-7.

[6] G.S. Beavers and D.D. Joseph. Boundary conditions at a naturally permeable wall. Journal of Fluid Mechanics, 30(01):197, oct 1967. doi: 10.1017/s0022112067001375.

[7] M. Bergmann and A. Iollo. Bioinspired swimming simulations. Journal of Computational Physics, 323: 310-321, 2016.

[8] W. P. Breugem and B. J. Boersma. Direct numerical simulations of turbulent flow over a permeable wall using a direct and a continuum approach. Physics of Fluids, 17(2):025103, feb 2005. doi: $10.1063 / 1.1835771$.

[9] W. P. Breugem, B. J. Boersma, and R. E. Uittenbogaard. The influence of wall permeability on turbulent channel flow. Journal of Fluid Mechanics, 562:35, aug 2006. doi: 10.1017/s0022112006000887.

[10] W.-P. Breugem, V. van Dijk, and R. Delfos. Flows through real porous media: X-ray computed tomography, experiments, and numerical simulations. Journal of Fluids Engineering, 136(4), feb 2014. doi: $10.1115 / 1.4025311$.

[11] Ch.-H. Bruneau. Boundary conditions on artificial frontiers for incompressible and compressible NavierStokes equations. M2AN, 34(2), 2000.

[12] Ch.-H. Bruneau and P. Fabrie. New efficient boundary conditions for incompressible Navier-Stokes equations : a well-posedness result. M2AN, 30(7), 1996.

[13] Ch.-H. Bruneau and K. Khadra. Highly parallel computing of a multigrid solver for 3d Navier-Stokes equations. J. of Computational Sc., 17(1), 2016.

[14] Ch.-H. Bruneau and M. Saad. The 2d lid-driven cavity problem revisited. Computers \& Fluids, 35(3), 2006.

[15] Ch.-H. Bruneau and S. Tancogne. Far field boundary conditions for incompressible flows computation. J. Appl. Anal. Comput., 8(3), 2018.

[16] M. Cieszko and J. Kubik. Derivation of matching conditions at the contact surface between fluidsaturated porous solid and bulk fluid. Transport in Porous Media, 34(1/3):319-336, 1999 . doi: 10.1023/a:1006590215455. 
[17] S. Ergun. Fluid flow through packed columns. Chem. Eng. Prog., 48:48-89, 1952.

[18] B. Hardy, J. De Wilde, and G. Winckelmans. A penalization method for the simulation of weakly compressible reacting gas-particle flows with general boundary conditions. Computers and Fluids, 190: 294-307, 2019.

[19] L. Isoardi, G. Chiavassa, G. Ciraolo, P. Haldenwang, E. Serre, Ph. Ghendrih, Y. Sarazin, F. Schwander, and P. Tamain. Penalization modeling of a limiter in the tokamak edge plasma. Journal of Computational Physics, 229(6):2220-2235, 2010.

[20] W. Jäger and A. Mikelić. Modeling effective interface laws for transport phenomena between an unconfined fluid and a porous medium using homogenization. Transport in Porous Media, 78(3):489-508, feb 2009. doi: $10.1007 / \mathrm{s} 11242-009-9354-9$.

[21] D. Jamet, M. Chandesris, and B. Goyeau. On the equivalence of the discontinuous one- and two-domain approaches for the modeling of transport phenomena at a fluid/porous interface. Transport in Porous Media, 78(3):403-418, dec 2008. doi: 10.1007/s11242-008-9314-9.

[22] T. Kajishima, S. Takiguchi, H. Hamasaki, and Y. Miyake. Turbulence structure of particle-laden flow in a vertical plane channel due to vortex shedding. JSME International Journal Series B, 44(4):526-535, 2001. doi: $10.1299 /$ jsmeb.44.526.

[23] G.H. Keetels, U. D'Ortona, W. Kramer, H.J.H. Clercx, K. Schneider, and G.J.F. van Heijst. Fourier spectral and wavelet solvers for the incompressible Navier-Stokes equations with volume-penalization: Convergence of a dipole-wall collision. Journal of Computational Physics, 227(2):919-945, 2007.

[24] N.K.-R. Kevlahan and J.-M. Ghidaglia. Computation of turbulent flow past an array of cylinders using a spectral method with Brinkman penalization. European Journal of Mechanics, B/Fluids, 20(3):333-350, 2001.

[25] K. Khadra, P. Angot, S. Parneix, and J.-P. Caltagirone. Fictitious domain approach for numerical modelling of Navier-Stokes equations. International Journal for Numerical Methods in Fluids, 34(8): 651-684, 2000. doi: 10.1002/1097-0363(20001230)34:8<651::aid-fld61>3.0.co;2-d.

[26] R. E. Larson and J. J. L. Higdon. Microscopic flow near the surface of two-dimensional porous media. part 1. axial flow. Journal of Fluid Mechanics, 166(-1):449, may 1986. doi: 10.1017/s0022112086000228.

[27] R. E. Larson and J. J. L. Higdon. Microscopic flow near the surface of two-dimensional porous media. part 2. transverse flow. Journal of Fluid Mechanics, 178(-1):119, may 1987. doi: $10.1017 / \mathrm{s} 0022112087001149$.

[28] D. Lasseux, A.A. Abbasian-Arani, and A. Ahmadi. On the stationary macroscopic inertial effects for one phase flow in ordered and disordered porous media. Phys. Fluids, 23:073-103, 2011.

[29] D. Lasseux, F.J. Valdés-Parada, and F. Bellet. Macroscopic model for unsteady flow in porous media. Journal of Fluid Mechanics, 862:283-311, 2019. doi: 10.1017/jfm.2018.878.

[30] C. Mimeau, G.-H. Cottet, and I. Mortazavi. Direct numerical simulations of three-dimensional flows past obstacles with a vortex penalization method. Computers and Fluids, 136:331-347, 2016.

[31] R. Mittal and G. Iaccarino. Immersed boundary methods. Annual Review of Fluid Mechanics, 37(1): 239-261, jan 2005. doi: 10.1146/annurev.fluid.37.061903.175743.

[32] G. Neale and W. Nader. Practical significance of Brinkman's extension of Darcy's law: Coupled parallel flows within a channel and a bounding porous medium. The Canadian Journal of Chemical Engineering, 52(4):475-478, aug 1974. doi: 10.1002/cjce.5450520407.

[33] J. A. Ochoa-Tapia and S. Whitaker. Momentum jump condition at the boundary between a porous medium and a homogeneous fluid: Inertial effects. Journal of Porous Media, 1(3):201-217, 1998. 
[34] J.A. Ochoa-Tapia and S. Whitaker. Momentum transfer at the boundary between a porous medium and a homogeneous fluid-I. theoretical development. International Journal of Heat and Mass Transfer, 38 (14):2635-2646, sep 1995. doi: 10.1016/0017-9310(94)00346-w.

[35] C. T. Paéz-García, F. J. Valdés-Parada, and D. Lasseux. Macroscopic momentum and mechanical energy equations for incompressible single-phase flow in porous media. Physical Review E, 95(2), feb 2017. doi: 10.1103 /physreve.95.023101.

[36] P. G. Saffman. On the boundary condition at the surface of a porous medium. Studies in Applied Mathematics, 50(2):93-101, jun 1971. doi: 10.1002/sapm197150293.

[37] A. Samanta, R. Vinuesa, I. Lashgari, P. Schlatter, and L. Brandt. Enhanced secondary motion of the turbulent flow through a porous square duct. Journal of Fluid Mechanics, 784:681-693, nov 2015. doi: $10.1017 / \mathrm{jfm} .2015 .623$.

[38] K. Schneider, S. Neffaa, and W.J.T. Bos. A pseudo-spectral method with volume penalisation for magnetohydrodynamic turbulence in confined domains. Computer Physics Communications, 182(1): $2-7,2011$.

[39] D. Shirokoff and J.-C. Nave. A sharp-interface active penalty method for the incompressible NavierStokes equations. Journal of Scientific Computing, 62(1):53-77, 2015.

[40] H.J. Spietz, M.M. Hejlesen, and J.H. Walther. Iterative Brinkman penalization for simulation of impulsively started flow past a sphere and a circular disc. Journal of Computational Physics, 336:261-274, 2017.

[41] K. Vafai. Convective flow and heat transfer in variable-porosity media. J. Fluid Mech., 147:233-259, 1984.

[42] F. J. Valdés-Parada, J. Alvarez-Ramírez, B. Goyeau, and J. A. Ochoa-Tapia. Computation of jump coefficients for momentum transfer between a porous medium and a fluid using a closed generalized transfer equation. Transport in Porous Media, mar 2009. doi: 10.1007/s11242-009-9370-9.

[43] F. J. Valdés-Parada, C. G. Aguilar-Madera, J. A. Ochoa-Tapia, and B. Goyeau. Velocity and stress jump conditions between a porous medium and a fluid. Advances in Water Resources, 62:327-339, dec 2013. doi: $10.1016 /$ j.advwatres.2013.08.008. 